\title{
Continuous In-Line Monitoring of Electrolyte and Urea Concentrations in Extracorporeal Circuits
}

\author{
Marc Berger ${ }^{1}$, Flora Sellering ${ }^{2}$, Hannes Röhrich ${ }^{2}$, Thorsten Perl' ${ }^{2}$, Hussam Mansour ${ }^{3}$, \\ Daniel Baasner ${ }^{4}$, Stefan Zimmermann ${ }^{1}$ \\ ${ }^{1}$ Leibniz University Hannover, Institute of Electrical Engineering and Measurement Technology, \\ Appelstr. 9A, 30167 Hannover, Germany \\ ${ }^{2}$ University Medical Center Göttingen, Robert-Koch-Str. 40, 37099 Göttingen, Germany \\ ${ }^{3}$ Barkey GmbH \& Co. KG, Gewerbestr. 8, 33818 Leopoldshöhe, Germany \\ ${ }^{4}$ InnoME GmbH, In der Tütenbeke 36, 32339 Espelkamp, Germany \\ Contact: berger@geml.uni-hannover.de
}

\section{Introduction}

The individualization of dialysis treatment is receiving more and more attention in research, because it can be beneficial particularly for critical ill patients in intensive care units (ICU) with acute kidney injury (AKI) [1-6].

AKI is understood as the rapid loss of the kidney's function. It is typically diagnosed by the accumulation of end products of the nitrogen metabolism such as urea nitrogen and creatinine in combination with reduced urine output [7]. AKI is often developed by critically ill patients with e.g. multi-organ failure or sepsis in ICU [8-10]. Such patients show a mortality rate of more than $50 \%$ [11]. The treatment of critical ill patients with AKI is usually the continuous renal replacement therapy (CRRT) [11-14]. The major difference between CRRT and the regular intermitted dialysis, applied to chronic kidney diseases, is the duration of treatment as well as the rate at which water and wastes are removed from the extracorporeal circuit via diffusion across the semipermeable membrane of the dialyzer [12]. While intermitted dialysis aims at a maximum clearance of waste products and normalization of electrolytes at a treatment duration of 3-4 hours, CRRT focuses on a significantly slower normalization of blood parameters over a treatment duration of several days, resulting in a benefit for critical ill patients, as a rapid change of osmotic substances can lead to different complications $[6,12,15]$.

For example, a rapid change in sodium plasma concentration, and thus an abrupt shift in plasma osmolarity, can cause cardiovascular instability, overhydrating of cells and disequilibrium syndrome with muscle cramps, fatigue symptoms and headaches. However, an insufficient sodium removal and hence sodium accumulation can lead to increased thirst, hypertension and pulmonary edema $[1,2,5,6]$.

Furthermore, a frequent complication of AKI is hyperkalemia with a particular potential for complications, since it can trigger heart arrhythmia. Similarly, hypokalemia can also cause arrhythmia. However, not only the absolute concentration is important for potassium. In $[1,16]$ it is shown, that an abrupt change in potassium concentration can trigger arrhythmias as well, because it induces a critical ratio between intracellular and extracellular potassium. Therefore, [17] recommends a maximum concentration gradient of $1.5 \mathrm{mmol} / \mathrm{l}$ between the dialysate and the blood with an exponential profile of the dialysate concentration to limit the diffusion and thus to limit the change of potassium concentration in the blood.

In order to adjust this required concentration gradient and to achieve the desired plasma concentration profile for potassium and sodium it is necessary to monitor the plasma concentration continuously.

In addition, ionized (free) calcium is another interesting parameter in extracorporeal circuits, since it is essential for blood coagulation. Especially in CRRT, anticoagulation is necessary to avoid clotting inside the dialyzer over the long period of treatment. As the critical ill patient may also be at increased risk of bleeding, a global anticoagulation using e.g. heparin, currently the most commonly used extracorporeal anticoagulant, has to be avoided. In contrast to heparin, citrate can be used as a regional anticoagulant. Therefore, citrate is added to the blood inside the extracorporeal circuit before the dialyzer, binding the ionized calcium as a complex and thus making the blood coagulation impossible. After the dialyzer, additional ionized calcium is added to the extracorporeal circuit to reactivate coagulation [18]. In order to control the exact amount of citrate being added, it is therefore necessary to continuously measure the ionized calcium concentration.

Furthermore, an important marker representing the efficiency of a dialysis treatment is the urea reduction ratio (URR) according to Eq. (1), as urea represents all waste products [19].

$U R R=\frac{U_{\text {pre }}-U_{\text {post }}}{U_{\text {pre }}} \cdot 100 \%$

Here, $U_{\text {pre }}$ is the pre-dialysis urea concentration in blood and $U_{\text {post }}$ is the blood urea concentration after 
the dialysis treatment. URRs less than $60 \%$ are associated with a higher mortality in patients during dialysis [20]. However, using Eq. (1) the efficiency can only be determined after treatment.

Thus, it is also beneficial to continuously monitor the blood urea concentration in order to evaluate the efficiency of dialysis treatment in-line and in real time.

The current practice determining blood parameters is to analyze blood samples in the laboratory or using a blood gas analyzer. This way, monitoring requires a considerable amount of personnel effort and costs. Moreover, the results are only available for a few discrete times with a time delay, making even quasi-continuously monitoring impossible.

Therefore, we presented in [21] a concept for continuous in-line monitoring of electrolyte concentration in extracorporeal circuits enabling an individualized dialysis treatment. Here, we used ion-selective electrodes (ISEs). Since it is difficult to achieve hemocompatibility with these sensors and direct in-line measurements in the blood are challenging, we have developed a concept in which the ISEs are separated from direct blood contact. As shown in Fig. 1 the ISEs are placed in a liquid-filled sensor compartment. This compartment is separated from the blood in the extracorporeal circuit by a dialysis membrane.

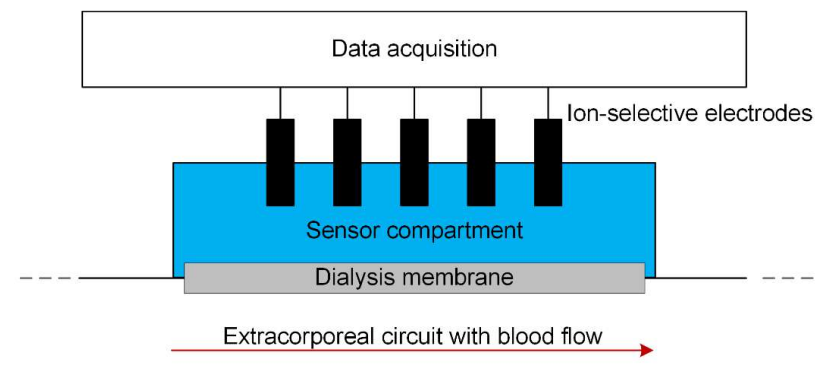

Fig. 1: Schematic depiction of the in-line measuring chamber.

The membrane allows all substances of interest, such as electrolytes and urea, to exchange between the sensor compartment and the blood. Proteins, for example, which can adsorb on the sensors surface and thus cause a sensor drift [3] or, in the worst case, blood coagulation, are retained by the membrane. Since substance transport between the blood and sensor compartment is based on diffusion, a certain time is required for a concentration equilibrium. A detailed investigation of the time-dependent behavior and the resulting errors of this measurement concept are shown in [22]. Fig. 2 shows a photo of the used measuring chamber consisting of two parts made of PEEK. The upper part contains the ISEs and has two Luer connectors for filling in the calibration solutions. The lower part also has two
Luer connectors, enabling a simple integration into the extracorporeal circuit. The semipermeable dialysis membrane is located between the two parts. The measuring chamber was developed and manufactured, particularly to achieve a hemocompatible blood flow, in cooperation with InnoMe $\mathrm{GmbH}$, 32339 Espelkamp, Germany and Barkey $\mathrm{GmbH}$ \& Co. KG, 33818 Leopoldshöhe, Germany.

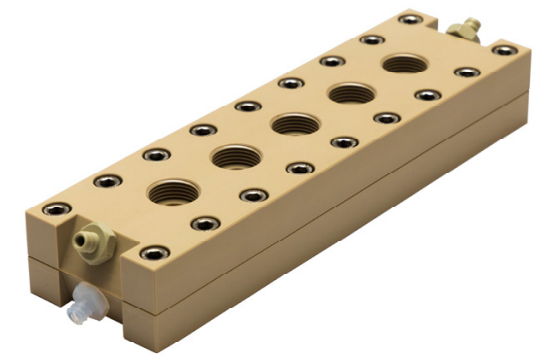

Fig. 2: Photo of the measuring chamber.

In this work, we present first measurements with this concept in an extracorporeal circuit using human packaged red blood cells (PRBC) as test medium to simulate realistic conditions.

\section{Setup of the Preclinical Investigation}

In order to simulate an extracorporeal circuit under realistic condition, we built up a test bench in cooperation with the University Medical Center Göttingen. Here, we used the multiFiltrate dialysis system and an AV 600S Ultraflux dialyzer from Fresenius Medical Care as depicted in Fig. 3.

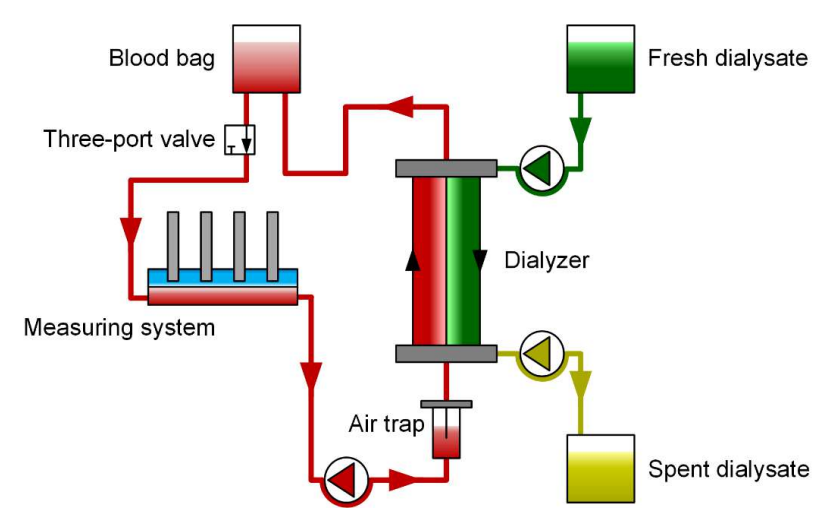

Fig 3: Schematic of the test bench using human packed red blood cells as test medium.

The PRBC are pumped from the blood bag through a tree-port valve, an air trap and the dialyzer by the dialysis system using peristaltic pumps with a flow rate of about $100 \mathrm{ml} / \mathrm{min}$, representing a typical flow rate in CRRT. The in-line measurement system is integrated into this simulated extracorporeal circuit. The three-port valve enables to draw blood samples, which are taken approximately every $10 \mathrm{~min}$ to be analyzed in a blood gas analyzer GEM Premier 
4000 from Instrumentation Laboratory as well as analyzed in the laboratory every $20 \mathrm{~min}$. On the other side of the filter membrane of the dialyzer, fresh dialysate is pumped into the dialyzer and the spent dialysate is pumped into a waste bag.

The electrolyte and urea concentrations in the fresh dialysate were varied for test purposes over the entire duration of the experiment. Diffusion between the dialysate and the blood caused a continuous change in the blood concentration, resulting in a maximum change of $50 \mathrm{mmol} / \mathrm{l}(113 \mathrm{mmol} / \mathrm{l}$ to $163 \mathrm{mmol} / \mathrm{l})$ for sodium, $8.8 \mathrm{mmol} / \mathrm{l}(1.9 \mathrm{mmol} / \mathrm{l}$ to $10.7 \mathrm{mmol} / \mathrm{l})$ for potassium, $1.695 \mathrm{mmol} / \mathrm{l}$ $(0.095 \mathrm{mmol} / /$ to $1.79 \mathrm{mmol} / \mathrm{l})$ for calcium and $178.2 \mathrm{mg} / \mathrm{dl}(1.8 \mathrm{mg} / \mathrm{dl}$ to $180 \mathrm{mg} / \mathrm{dl})$ for blood urea nitrogen (BUN), covering the clinical relevant concentration range.

\section{Concept of the Urea Sensor}

To measure urea, we use a biosensor consisting of an ammonium ISE and the enzyme urease. Using urease, urea is catalyzed into ammonium and carbonate ions according to Eq. (2) [23], while the ammonium ions are measured with an ammonium ISE.

$\mathrm{CO}\left(\mathrm{NH}_{2}\right)_{2}+2 \mathrm{H}_{2} \mathrm{O} \rightarrow 2 \mathrm{NH}_{4}^{+}+\mathrm{CO}_{3}^{2-}$

There are many approaches to immobilize the enzyme near the electrodes, e.g. cross-linking of the enzyme [24]. However, such approaches often use toxic chemicals making an in-line measurement difficult. Moreover, the activity of the enzyme is often reduced. Therefore, we use a simple approach for immobilization, consisting of a dialysis membrane (RCT NatureFlex-NP from Reichelt Chemietechnik) with a molecular weight cutoff of $10 \mathrm{kDa}-20 \mathrm{kDa}$ retaining the enzyme solution inside a small enzyme compartment created by a $0.5 \mathrm{~mm}$ spacer, which is bonded to the ISE, as depicted in Fig. 4. The bonding of the $0.5 \mathrm{~mm}$ spacer to the ISE was realized in cooperation with InnoMe $\mathrm{GmbH}, 32339$ Espelkamp, Germany.

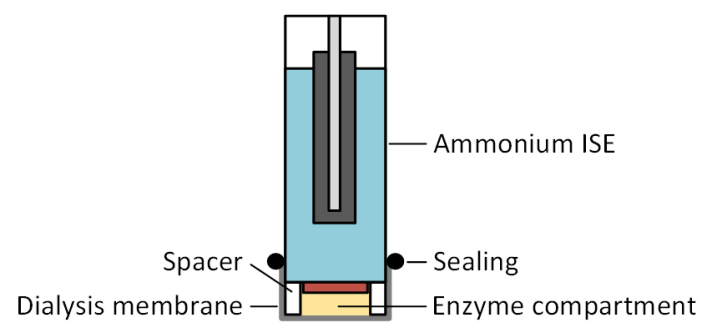

Fig 4: Schematic cross-section of the urease sensor consisting of an ammonium ISE as well as a $0.5 \mathrm{~mm}$ spacer and a dialysis membrane forming the enzyme compartment.
Since urea has a molecular weight of about $60 \mathrm{Da}$ it can diffuse inside the enzyme compartment. Urease, however, cannot pass the membrane and is retained inside this compartment, as its molecular weight is $480 \mathrm{kDa}$. Thus, the ammonium ions are generated near the sensitive layer of the ammonium ISE. The urea sensor was integrated into the sensor compartment of the measuring chamber.

\section{Results of the Electrolyte Monitoring}

During the investigation, we changed the blood concentration of all parameters (sodium, potassium, calcium and urea) simultaneously, as described above, via the dialysate concentration in the clinically relevant range. As a result, it is possible to investigate the cross-sensitivity of the ISEs to other ions. The ISEs were previously calibrated by filling the sensor compartment with two different calibration solutions, neglecting the cross-sensitivities. Both have different concentrations of the different electrolytes but the same ionic strength of $160 \mathrm{mmol} / \mathrm{l}$, which is comparable to blood ionic strength enabling a so-called concentration calibration in which the output voltage of the respective electrodes vs. a Ag$\mathrm{AgCl}$ reference electrode in a certain concentration range is approximately proportional to the concentration and not only to the activity of the ions [25].

Fig. 5 shows the results for sodium over a period of 8 hours. The circles are the discrete blood samples analyzed by the blood gas analyzer used as reference. The solid curve is the output signal of the calibrated electrode. It can be seen that there is a deviation between the sensor and the reference at the beginning of the measurement. This is due to the calibration solution still in the sensor compartment when integrating the system into the extracorporeal circuit and having different initial electrolyte concentrations compared to blood. Thus, a certain time is required until the concentration equilibration between the blood and the sensor compartment is reached.

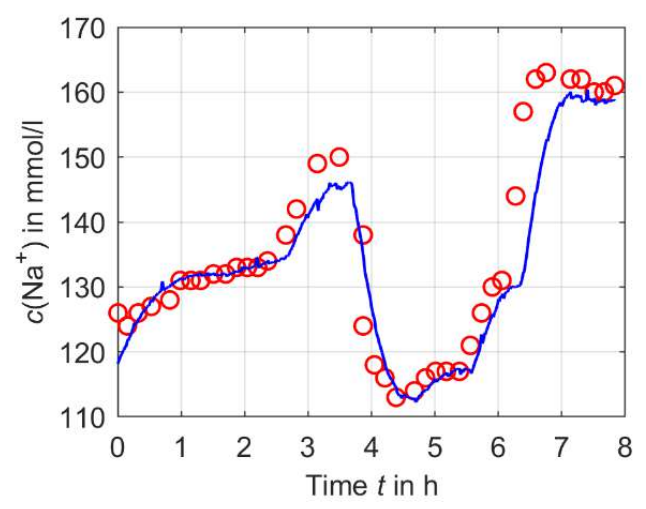

Fig. 5: Continuous measured sodium concentration (solid line) compared to the blood gas analyzer as reference (circles). 
After this initial mismatch, the measured sensor signal and reference signal correlate very well. Just at about 3.5 hours and 6.5 hours, there are small deviations. This is due to the dynamic behavior of the measurement concept as shown in [22]. However, the blood concentration was changed that fast for test purpose, way off physiological rates.

For potassium, depicted in Fig. 6, there is also an initial discrepancy of the sensor signal due to the initial filling of the sensor compartment with the calibration solution. Again, the sensor signal and the reference signal agree perfectly for most of the time after the initial mismatch.

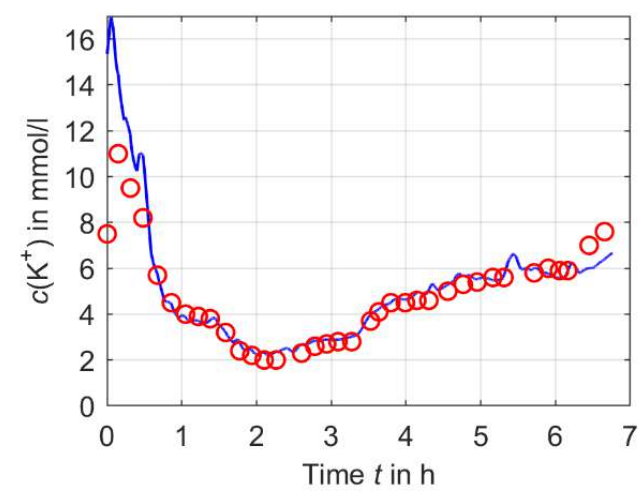

Fig 6: Continuous measured potassium concentration (solid line) compared to the blood gas analyzer as reference (circles).

As shown in Fig. 7, no initial deviation can be observed with calcium, since the blood concentration and concentration of the calibration solution coincidentally matched. With the exception of hours 6 and 7 , where a small deviation of about $0.25 \mathrm{mmol} / \mathrm{l}$ can be seen, the reference and the measurement signal agree. This also applies for the very low concentration after 7.5 hours, which is particularly interesting for citrate anticoagulation.

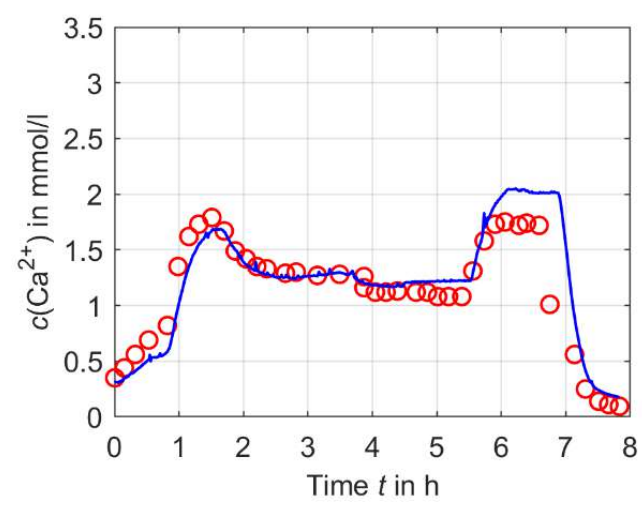

Fig 7: Continuous measured calcium concentration (solid line) compared to the blood gas analyzer as reference (circles).

The results show that the respective electrolytes can be measured in good agreement with the refer- ence system. No relevant sensor drift or crosssensitivity could be observed during the investigation.

\section{Results of the Urea Monitoring}

The urea sensor was not calibrated in advance, but in the aftermath using two different laboratory values, which were taken about every $20 \mathrm{~min}$ and are the reference values for the urea sensor.

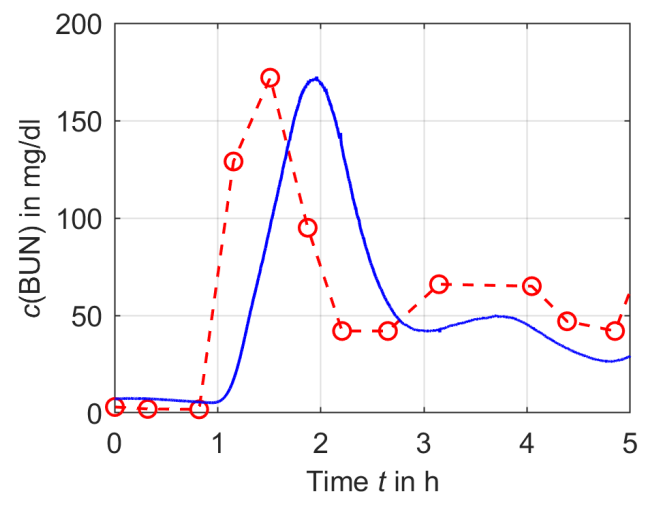

Fig 8: Continuous measured urea concentration (solid line) compared to the laboratory analysis (circles).

The results of the laboratory analysis for blood urea nitrogen (BUN) are depicted in Fig. 8 as circles. The measured curve of the urea sensor is shown as solid line. It can be seen that there is a time delay of about half an hour between the reference value and the measured value, due to the additional membrane and diffusion distance to reach the enzyme compartment of the urea sensor. In addition, a drift of the sensor signal can be observed. One possible reason could be a loss of the enzyme urease from the enzyme compartment due to a damaged membrane. Therefore, in this first test, the sensor provides only a trend of BUN until about 5 hours after starting the measurement. Further investigations are required to exclude any possible errors in the immobilization process.

\section{Conclusion}

In this work, we have investigated the developed measurement system for continuous in-line measurement of electrolyte and urea concentrations under realistic conditions in a preclinical study using human packaged red blood cells as test medium in an extracorporeal circuit.

It is shown that the measured sodium, potassium and calcium concentrations match very well with the blood gas analyzer values used as a reference system. No relevant sensor drift or cross-sensitivity to other ions could be observed over a test duration of up to 8 hours. 
The urea sensor, consisting of an ammonium ISE and the enzyme urease, immobilized hemocompatibly using a dialysis membrane in front of the electrode, showed a drift of the sensor signal. Possibly caused by a defect in the enzyme compartment membrane.

In conclusion, the presented in-line measuring system could be a considerable advance compared to the current Gold Standard, where only a few discrete measurements per dialysis treatment are obtained and the measured values are available with a time delay due to laboratory analysis. Personnel effort is also significantly reduced. The continuous measurement of blood parameters is an important step towards individualized dialysis, leading to a significant improvement for the treatment of the patients.

\section{Acknowledgments}

Supported by the German Federal Ministry of Education and Research (BMBF) under the grant 13GW0085B.

\section{References}

[1] B. F. Palmer, Individualizing the Dialysate in the Hemodialysis Patient, Seminars in Dialysis 14, 41-49 (2001); doi: 10.1046/j.1525-139x.2001.00013.x.

[2] F. Locatelli, V. La Milia, L. Violo, L. Del Vecchio, S. Di Filippo, Optimizing haemodialysate composition, Clinical kidney journal 8, 580-589 (2015); doi: 10.1093/ckj/sfv057.

[3] M. K. Sharma, F. P. Wieringa, A. J. H. Frijns, J. P. Kooman, On-line monitoring of electrolytes in hemodialysis: on the road towards individualizing treatment, Expert review of medical devices 13, 933-943 (2016); doi: 10.1080/17434440.2016.1230494.

[4] C. P. Kovesdy, D. L. Regidor, R. Mehrotra, J. Jing, C. J. McAllister, S. Greenland, J. D. Kopple, K. KalantarZadeh, Serum and dialysate potassium concentrations and survival in hemodialysis patients, Clinical journal of the American Society of Nephrology : CJASN 2, 999-1007 (2007); doi: 10.2215/CJN.04451206.

[5] F. M. de Paula, A. J. Peixoto, L. V. Pinto, D. Dorigo, P. J. M. Patricio, S. F. F. Santos, Clinical consequences of an individualized dialysate sodium prescription in hemodialysis patients, Kidney international 66, 1232-1238 (2004); doi: 10.1111/j.15231755.2004.00876.x.

[6] S. Stiller, E. Bonnie-Schorn, A. Grassmann, I. Uhlenbusch-Körwer, H. Mann, A Critical Review of Sodium Profiling for Hemodialysis, Seminars in Dialysis 14, 337-347 (2001); doi: 10.1046/j.1525139X.2001.00086.x.

[7] R. Bellomo, J. A. Kellum, C. Ronco, Acute kidney injury, The Lancet 380, 756-766 (2012); doi: 10.1016/S0140-6736(11)61454-2.

[8] A. Zarjou, A. Agarwal, Sepsis and acute kidney injury, Journal of the American Society of Nephrology : JASN 22, 999-1006 (2011); doi: 10.1681/ASN.2010050484.
[9] A. E. Baue, E. Faist, D. E. Fry, Multiple Organ Failure: Pathophysiology, Prevention, and Therapy. Springer New York, New York; 2000.

[10] S. M. Bagshaw, C. George, R. Bellomo, Early acute kidney injury and sepsis: A multicentre evaluation, Critical care (London, England) 12, R47 (2008); doi: 10.1186/cc6863.

[11] Z. Ricci, R. Bellomo, C. Ronco, Dose of dialysis in acute renal failure, Clinical journal of the American Society of Nephrology : CJASN 1, 380-388 (2006); doi: $10.2215 /$ JJN.00520705.

[12] P. Patel, V. Nandwani, P. J. McCarthy, S. A. Conrad, L. Keith Scott, Continuous renal replacement therapies: A brief primer for the neurointensivist, Neurocritical care 13, 286-294 (2010); doi: 10.1007/s12028010-9386-6.

[13] D. Patschan, G. A. Müller, Acute kidney injury, Journal of injury \& violence research 7, 19-26 (2015); doi: 10.5249/jivr.v7i1.604

[14] J. Tumlin, R. Wali, W. Williams, P. Murray, A. J. Tolwani, A. K. Vinnikova, H. M. Szerlip, J. Ye, E. P. Paganini, L. Dworkin, K. W. Finkel, M. A. Kraus, H. D. Humes, Efficacy and safety of renal tubule cell therapy for acute renal failure, Journal of the American Society of Nephrology : JASN 19, 1034-1040 (2008); doi: 10.1681/ASN.2007080895.

[15] M. Sivalingam, K. Farrington, Haemodialysis, Medicine 35, 461-465 (2007); doi: 10.1016/j.mpmed.2007.05.005.

[16] M. Buemi, E. Aloisi, G. Coppolino, S. Loddo, E. Crascì, C. Aloisi, A. Barillà, V. Cosentini, L. Nostro, C. Caccamo, F. Floccari, A. Romeo, N. Frisina, D. Teti, The effect of two different protocols of potassium haemodiafiltration on QT dispersion, Nephrology, dialysis, transplantation : official publication of the European Dialysis and Transplant Association - European Renal Association 20, 1148-1154 (2005); doi: 10.1093/ndt/gfh770.

[17] B. Redaelli, F. Locatelli, D. Limido, S. Andrulli, M. G. Signorini, S. Sforzini, L. Bonoldi, A. Vincenti, S. Cerutti, G. Orlandini, Effect of a new model of hemodialysis potassium removal on the control of ventricular arrhythmias, Kidney international 50, 609-617 (1996).

[18] A. Davenport, A. Tolwani, Citrate anticoagulation for continuous renal replacement therapy (CRRT) in patients with acute kidney injury admitted to the intensive care unit, NDT plus 2, 439-447 (2009); doi: 10.1093/ndtplus/sfp136.

[19] P. R. Keshaviah, J. P. Ebben, P. F. Emerson, On-line monitoring of the delivery of the hemodialysis prescription, Pediatric Nephrology 9, S2-S8 (1995); doi: 10.1007/BF00867675.

[20] W. F. Owen, N. L. Lew, Y. Liu, E. G. Lowrie, J. M. Lazarus, The urea reduction ratio and serum albumin concentration as predictors of mortality in patients undergoing hemodialysis, The New England journal of medicine 329, 1001-1006 (1993); doi: 10.1056/NEJM199309303291404.

[21] M. Berger, S. Zimmermann, eds., P1.03 - In-lineMonitoring der Elektrolyt- und Harnstoffkonzentration in extrakorporalen Kreisläufen zur Individualisierung der Nierenersatztherapie; 2017.

[22] M. Berger, M. Faulstich, T. Perl, S. Zimmermann, Continuous in-line monitoring of electrolyte concentrations in extracorporeal circuits for individualization of dialysis treatment, Journal of Sensors and Sensor 
Systems 7, 559-567 (2018); doi: 10.5194/jsss-7-5592018.

[23] G. Hu, J. A. Pojman, S. K. Scott, M. M. Wrobel, A. F. Taylor, Base-catalyzed feedback in the urea-urease reaction, The journal of physical chemistry. B 114, 14059-14063 (2010); doi: 10.1021/jp106532d.

[24] M. Singh, N. Verma, A. Garg, N. Redhu, Urea biosensors, Sensors and Actuators B: Chemical 134, 345-351 (2008); doi: 10.1016/j.snb.2008.04.025.

[25] K. Cammann, H. Galster, Das Arbeiten mit ionenselektiven Elektroden: Eine Einführung für Praktiker. Springer Berlin Heidelberg, Berlin, Heidelberg; 1996. 